\title{
Dynamic conductance of mesoscopic waveguides
}

\author{
Man-Kit Yip, Wing-Chung Kwok, and Jian Wanga) \\ Department of Physics, The University of Hong Kong, Pokfulam Road, Hong Kong, China \\ Hong Guo \\ Centre for the Physics of Materials and Department of Physics, McGill University, \\ Montreal, PQ H3A 2T8, Canada
}

(Received 7 August 2000; accepted for publication 8 November 2000)

\begin{abstract}
We report a theoretical investigation of dynamic conductance $G(\omega)$, for general ac frequency $\omega$, of two-dimensional mesoscopic waveguides whose transport is characterized by antiresonances. We calculate $G(\omega)$ by numerically evaluating nonequilibrium Green's functions. By tuning the ac frequency we observe photon-assisted resonant transport as well as a gradual smearing out of the antiresonances. The antiresonance causes the dynamic response to vary between capacitive-like behavior to that of the inductive-like behavior. (ㅇ) 2001 American Institute of Physics.
\end{abstract}

[DOI: $10.1063 / 1.1337086]$

\section{INTRODUCTION}

Transport properties of two-dimensional (2D) mesoscopic electron waveguides have been studied extensively since the development of controlled crystal growth and lithographic techniques. ${ }^{1-5}$ So far, coherent electron conduction under a small dc bias is well understood both theoretically and experimentally for these systems. However, transport properties of mesoscopic conductors under an ac bias still present many open questions, and this subject is increasingly attracting more attention. ${ }^{6-11}$ Under ac fields the dynamic conductance $G(\omega)$ consists not only of particle current, but also of displacement current. ${ }^{11}$ To incorporate this effect into a quantum transport theory, one necessarily needs to solve the transport problem in conjunction with electrodynamics. ${ }^{12}$ ac transport theory including electrodynamic effects has been developed from a scattering matrix theory (SMT) point of view $^{11}$ and applied ${ }^{13}$ for analyzing 2D mesoscopic conductors to first order in ac frequency $\omega-$ a situation for which $\omega$ is small. In order to gain physical insight into the dynamic response of mesoscopic waveguides at general ac frequency $\omega$ and to construct a general physical picture of quantum conduction under dynamic conditions, in this article we report on investigations of dynamical conductance at finite frequency for a class of 2D electron waveguides, i.e., we report the behavior of $G(\omega)$ beyond the $\omega \rightarrow 0$ limit.

To be specific, we have investigated dynamic conductance for two electron waveguides which are represented by the 2D model shown in the inset of Fig. 2. The width of leads is $W$, and by adjusting the values of two potential barriers $V_{a}$ and $V_{b}$, we obtain two different 2D wires: (1) $V_{a}=\infty$ and $V_{b}=0$; this corresponds to a T-shaped waveguide ${ }^{14}$ with the length of stub equal to $2 W$; (2) $V_{a}=0$ and $V_{b}=\infty$; this corresponds to an $L$-shaped quantum dot. ${ }^{15}$ For these $2 \mathrm{D}$ open waveguides, the electron scattering region is strongly coupled to the leads, thus they are usually transmissive for electrons coming from one lead and going to another. It is

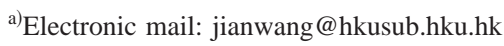

known ${ }^{16}$ that there are quantum bound states localized around the scattering region of these systems. The existence of potential barriers somewhat isolates the scattering region, thereby pushing the quantum bound states into the continuum and resulting in resonant tunneling. ${ }^{15}$ The quasibound states of these systems are also quite different from those of one-dimensional (1D) double-barrier resonant tunneling structures: instead of a complete transmission at resonances in 1D, 2D waveguides are characterized by an antiresonant state ${ }^{17,18}$ corresponding to a complete reflection of incoming electrons. Recently, a general theory has been formulated to consider conductance of molecular wires and a type of antiresonance was predicted. ${ }^{19}$ We focus on the behavior of dynamic conductance $G(\omega)$ both off and at antiresonance of these 2D waveguides. Our calculation is based on evaluating nonequilibrium Green's functions (NEGF) using the ac transport theory of Ref. 20 which allows us to examine the importance of the displacement current. An analytic expression of dynamic conductance in the wideband limit has been derived for the situation near an antiresonant state. For more general situations we numerically compute various quantities of interest. Due to the ac field, electrons traversing the scattering region can absorb and emit photons leading to photon-assisted transport. By tuning the ac frequency $\omega$ we observe a gradual smearing out of the antiresonances. Interesting capacitive-like and inductive-like behavior is observed.

The article is organized as follows: the theoretical and numerical methods used for this investigation are presented in Sec. II, Sec. III presents results, and Sec. IV is a discussion and summary of results.

\section{THEORETICAL AND NUMERICAL METHODS}

Our investigation on the 2D waveguides is based on the theoretical formulation of NEGF, ${ }^{20}$ where the displacement current is partitioned among the leads through a gauge invariance condition. ${ }^{21}$ Since the details of this formalism can be found in Ref. 20, we refer interested readers to that ref- 
erence for mathematical details. In the following we only outline the necessary formulas which are needed for our numerical analysis of this work.

Within the NEGF formalism ${ }^{20}$ the dynamic conductance $G_{\alpha \beta}(\omega)$ between leads labeled by $\alpha$ and $\beta$ is

$$
G_{\alpha \beta}=G_{\alpha \beta}^{c}-G_{\beta}^{d} \frac{\Sigma_{\gamma} G_{\alpha \gamma}^{c}}{\sum_{\gamma} G_{\gamma}^{d}} .
$$

In this expression, the quantity with superscript $d$ is the conductance coefficient contributed by the displacement current, while that with superscript $c$ is contributed by the particle current. The coefficients are given by ${ }^{20}$

$$
G_{\beta}^{d}(\omega)=-q \omega \int \frac{d E}{2 \pi} \operatorname{Tr}\left[\bar{g}_{\beta}^{<}\right],
$$

and

$$
\begin{aligned}
G_{\alpha \beta}^{c}(\omega)= & -q \int \frac{d E}{2 \pi} \operatorname{Tr}\left[\bar{g}_{\beta}^{<}\left(\Sigma_{0 \alpha}^{a}-\bar{\Sigma}_{0 \alpha}^{r}\right)+\bar{g}_{\beta}^{r} \Sigma_{0 \alpha}^{<}-\bar{\Sigma}_{0 \alpha}^{<} \bar{g}_{\beta}^{a}\right. \\
& \left.+\left(\bar{G}_{0}^{r} \bar{\sigma}_{\alpha}^{<}-\bar{\sigma}_{\alpha}^{<} G_{0}^{a}+\bar{G}_{0}^{<} \bar{\sigma}_{\alpha}^{a}-\bar{\sigma}_{\alpha}^{r} G_{0}^{<}\right) \delta_{\alpha \beta}\right],
\end{aligned}
$$

where $q$ is the electron charge. Before defining the other quantities in these equations, we emphasise that inclusion of the displacement current contribution preserves current conservation under ac condition, and expression (1) also satisfies gauge invariance. These facts are mathematically expressed $\operatorname{as}^{22} \Sigma_{\alpha} G_{\alpha \beta}=\Sigma_{\beta} G_{\alpha \beta}=0$.

Quantities $\sigma_{\alpha}^{r, a,<}$ and $\bar{g}_{\alpha}^{r, a,<}$ in Eq. (3) are correction terms to equilibrium quantities $\Sigma_{0 \alpha}^{r, a,<}$, and $\bar{G}_{0}^{r, a,<}$, and superscripts $r, a,<$ refer to retarded, advanced and lesser quantities in the usual language of Green's functions. In our notation $^{20} g \equiv g(E, E)$ and $\bar{g} \equiv \bar{g}(E+\hbar \omega, E)$, which are double time Fourier transforms of the Green's function $g\left(t, t^{\prime}\right)$. The Green's functions in Eqs. (2) and (3) are given by $^{20,23,24}$

$$
\begin{aligned}
& \bar{g}_{\alpha}^{r, a}=\bar{G}_{0}^{r, a} \bar{\sigma}_{\alpha}^{r, a} G_{0}^{r, a}, \\
& \bar{g}_{\alpha}^{<}=\bar{G}_{0}^{r} \bar{\sigma}_{\alpha}^{<} G_{0}^{a}+\bar{G}_{0}^{r} \bar{\sigma}_{\alpha}^{r} G_{0}^{<}+\bar{G}_{0}^{<} \bar{\sigma}_{\alpha}^{a} G_{0}^{a}, \\
& \bar{\sigma}_{\alpha}^{r, a,<}=\frac{q}{\omega}\left[\Sigma_{0 \alpha}^{r, a,<}-\bar{\Sigma}_{0 \alpha}^{r, a,<}\right] .
\end{aligned}
$$

In these equations, $G_{0}^{<}=G_{0}^{r} \Sigma_{0}^{<} G_{0}^{a}$ is the equilibrium lesser Green's function and $\Sigma_{0}^{<}=-f\left[\Sigma^{r}-\Sigma^{a}\right] \equiv i f \Gamma$, where $f$ is the Fermi-Dirac distribution. Equation (5) is simply the generalization of the Langreth theorem while dealing with the Dyson equation. The quantity $\bar{\sigma}_{\alpha}^{<}$in Eq. (6) can be rewritten $^{20}$ as $\bar{\sigma}_{\alpha}^{<}=(i q)\left[\Gamma_{\alpha} f-\bar{\Gamma}_{\alpha} \bar{f}\right] / \omega$.

The earlier definitions of various quantities together with Eq. (1) give the necessary expressions for evaluation of the dynamic conductance of any mesoscopic conductor, and this formulation includes the contribution from electrodynamics. Furthermore, Eq. (1) is a general result: it is suitable for analyzing ac transport coefficients in general terms of frequency $\omega$ for systems far from equilibrium. In the rest of this section, we briefly outline the numerical procedure which is used in this work to calculate the most essential quantities
$G_{0}^{r}$ and $\Sigma^{r}$. Once they are obtained, all expressions of the earlier formalism are then evaluated in a straightforward manner.

Following the tight-binding approach of Ref. 25, we discretize the waveguides of the inset of Fig. 2 using a 2D uniform mesh, in which hopping between nearest neighbor sites is considered. The effect of coupling the waveguides to semi-infinitely long leads is accounted for by introducing the self-energy $\Sigma^{r}$. The equilibrium retarded Green's function $G_{0}^{r}$ is then calculated from its definition

$$
G_{0}^{r}=\left[E I-H_{c}-\Sigma^{r}\right]^{-1},
$$

where $H_{c}$ is the tight-binding Hamiltonian of the scattering region. In our model (see inset of Fig. 2), the leads are perfect $2 \mathrm{D}$ wires which extend far away from the waveguides. It is relatively easy to prove, ${ }^{25,26}$ for this model of the lead, that the self-energy $\Sigma^{r}$ is just given by the Green's function of a semi-infinite $2 \mathrm{D}$ pipe. For lead $p$, the self-energy is thus given by ${ }^{26}$

$$
\sum_{p}^{r}(i, j)=t \sum_{m \in p} \chi_{m}\left(p_{i}\right) \exp \left[i k_{m} a\right] \chi_{m}\left(p_{j}\right),
$$

where $i, j$ label lattice sites located inside the scattering region (the waveguides of inset of Fig. 2) but adjacent to the interface connecting to lead $p . \chi_{m}$ is the transverse wave function of the leads of mode $m$ and for our model they are simply sine functions; $t$ denotes a coupling constant which is essentially given by the lattice constant. ${ }^{27}$ The sum is over all states including evanescent states, out of which the positive evanescent states (so that $i k_{m}<0$ ) are used for convergence. After $\Sigma_{p}^{r}$ is calculated, the total self-energy due to coupling to all the leads is obtained by $\Sigma^{r}=\Sigma_{p} \Sigma_{p}^{r}$.

In general, $\Sigma^{r}$ is a symmetric matrix with nonzero elements at positions corresponding to the interface sites between a lead and the scattering region. Because evaluating $G_{0}^{r}$ from Eq. (7) corresponds to the inversion of a matrix, a reasonable numbering scheme to the lattice sites is to minimize the bandwidth of the matrix and thus reduce the numerical computation. For example, to obtain the narrowest bandwidth for the T-shaped waveguide we label each site sequentially along the transverse direction of a particular probe. As a result, the self-energy due to this probe becomes a block diagonal matrix. All nonzero matrix elements correspond to the sites along the interface of the waveguide and the lead. For a T-shaped waveguide, the bandwidth of the self-energy matrix is about twice the number of discretized points along the transverse direction of the stub $(2 W)$. Therefore, the longer the stub, the larger the bandwidth.

For completeness, we provide here all the relevant details of quantities for our calculation

$$
\begin{aligned}
& \hbar \nu_{n}=-2 a t \sin \left(k_{x, n} a\right), \\
& k_{x, n} a=\cos ^{-1}\left[\left(E-\epsilon_{n, 0}\right) /(2 t)+1\right], \\
& \chi_{n}(j)=\sqrt{\frac{2}{N+1}} \sin \left(\frac{n \pi j}{N+1}\right),
\end{aligned}
$$




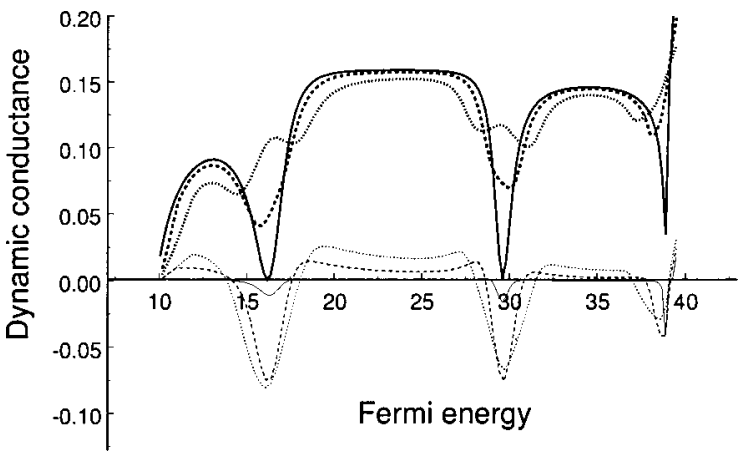

FIG. 1. Dynamic conductance versus Fermi energy for a 2D T-shaped waveguide. The results are obtained by using the NEGF.

$$
\begin{aligned}
& \epsilon_{n, 0}=2 t\left[\cos \left(\frac{n \pi}{N+1}\right)-1\right], \\
& E=2 t\left[\cos \left(k_{x, n} a\right)+\cos \left(\frac{n \pi}{N+1}\right)-2\right],
\end{aligned}
$$

where $a, v_{n}$ and $k_{x, n}$ denote the tight-binding lattice constant, longitudinal velocity and longitudinal wave vector, respectively. $\epsilon_{n, 0}$ and $E$, represent the $n$th band energy of the electrons in the leads and the total energy of the electrons respectively. Finally, using the Green's function one can also calculate the dc conductance from the scattering matrix $s$ through the discretized version of the Fisher-Lee relation

$s_{n m q p}=-\delta_{n m q p}+\frac{i \hbar \sqrt{v_{n} v_{m}}}{a} \sum_{j \in q} \sum_{i \in p} \chi_{n}(j) G_{0}^{r}(j, i) \chi_{m}(i)$,

where $n, m$ are the propagating mode numbers of leads $q$ and $p$, respectively.

Using the NEGF formalism and the numerical procedures outlined in this section, we calculated dynamic conductance for the electron waveguides of the inset of Fig. (2) and the results are presented in the next section. From hereon, we fix units using $\hbar=a=2 m=1$. In this system of units, for a wire of width $W=500 \AA$ and using effective mass $m$ corresponding to GaAs, $\omega=1$ corresponds to 3.4 $\times 10^{11} \mathrm{~Hz}$.

\section{RESULTS}

The dynamic conductance $G_{\alpha \beta}(\omega)$ is a complex quantity which has two components. Its real part, denoted as $G_{R}$, characterizes the dissipative component, whereas the imaginary part, denoted $G_{I}$, represents the nondissipative component. At small frequencies, one can expand $G_{\alpha \beta}(\omega)$ in terms of $\omega$ to obtain ${ }^{28} G_{\alpha \beta}(\omega)=G_{\alpha \beta}(0)-i \omega E_{\alpha \beta}+O\left(\omega^{2}\right)$ where $E_{\alpha \beta}$ is called the emittance. In SMT, $E_{\alpha \beta}$ has been analyzed in detail ${ }^{29}$ and it is found to be related to the local density of states characterizing the quantum scattering process. ${ }^{29}$ For higher frequencies, which is the concern of this work, we use Eq. (1) to predict dynamic conductance for a general $\omega$.

$G_{11}(\omega)$ of the T-shaped waveguide at three different frequencies is shown in Fig. (1) as a function of the scattering electron energy for the range corresponding to the first subband, obtained from NEGF formalism outlined in the previ- ous section. The upper three lines show the dissipative component $G_{R}$, while the lower three lines represent the nondissipative component $G_{I}$. The frequencies are $\omega=0.1$, 1.0, and 2.0, from top to bottom, respectively. For the smallest frequency $\omega=0.1, G_{R} \equiv \operatorname{Re}\left[G_{11}(\omega)\right]$ is very close to the dc conductance directly obtained from the scattering matrix ${ }^{13}$ while $G_{I}$ is very small. This is reasonable because when $\omega$ $\rightarrow 0$, we indeed expect $G_{R} \sim G_{11}(0)$ while $G_{I} \sim-\omega E_{11}$. Similar behavior is observed for both waveguides at small $\omega$.

Since the waveguides are very transmissive, their dc transmission coefficient is in general close to unity (for one subband). At a certain resonance point corresponding to quasibound states we get complete reflection, corresponding to antiresonance. ${ }^{17,18}$ The anti resonance behavior is clearly observed in the curve of $G_{R}$ in Fig. (1) at frequency $\omega=0.1$, as indicated by the minima of $G_{R}$. For most energies, the nondissipative component $G_{I}$ is positive, indicating an inductive-like dynamic response of the system. However, it is negative near the antiresonant points: for the range of the first subband energy there are three negative dips in $G_{I}$ (see Fig. 1), showing a capacitive-like response at these energies. The capacitive behavior is consistent with the anti-resonant picture, e.g., at an antiresonance there is no dc conduction, thus the system behaves like a capacitor. The capacitive-like behavior of $G_{11}$ is mainly due to the internal dynamic response which results from Coulomb interactions. As the frequency $\omega$ is increased, the dynamic conductance changes significantly near antiresonance points, as shown in Fig. 1. In particular, we note that the sharp minimum of $G_{R}$ is smeared out by $\omega$, indicating a decrease of dynamic resistance. Another result is that when $\omega$ is increased, both inductive-like and capacitive-like behavior are enhanced.

The behavior of $G_{R}$ and $G_{I}$ as $\omega$ is increased can be qualitatively understood from a classical circuit model. ${ }^{30}$ Due to both inductive and capacitive responses of these waveguides, one can consider our system as an inductor in series with a parallel connection of a capacitor and a resistor. For this classical circuit the dynamic conductance can be written $^{30}$ in the following form up to second order in frequency $\omega$ :

$G(\omega)=(1 / R)-i \omega\left[C-L / R^{2}\right]+\omega^{2}(L / R)\left[2 C-L / R^{2}\right]$

The linear term in $\omega$, which corresponds to the nondissipative part of dynamic conductance $G_{I}$, exhibits a competition between two different dynamic responses. If $C>L / R^{2}$, the response is capacitive-like with a negative $G_{I}$, and $\left|G_{I}\right|$ increases linearly with $\omega$ at small frequencies, which is consistent with Fig. 1. A similar argument for $G_{I}$ applies to the inductive-like region when $C<L / R^{2}$. The transition from capacitive-like to inductive-like behavior occurs when $C$ $=L / R^{2}$, when $G_{I}$ vanishes to second order in $\omega$. On the other hand, the dissipative component $G_{R}$, near antiresonant points, can increase or decrease with $\omega^{2}$ depending on the sign of the second-order term in Eq. (11). Our data of Fig. 1 indicates an increase of $G_{R}$ at antiresonance, hence, for this waveguide the effective parameter $C>L /\left(2 R^{2}\right)$. Note, however, that when the frequency is so large that $\omega^{3}$ or higher order terms cannot be neglected, Eq. (11) breaks down. 


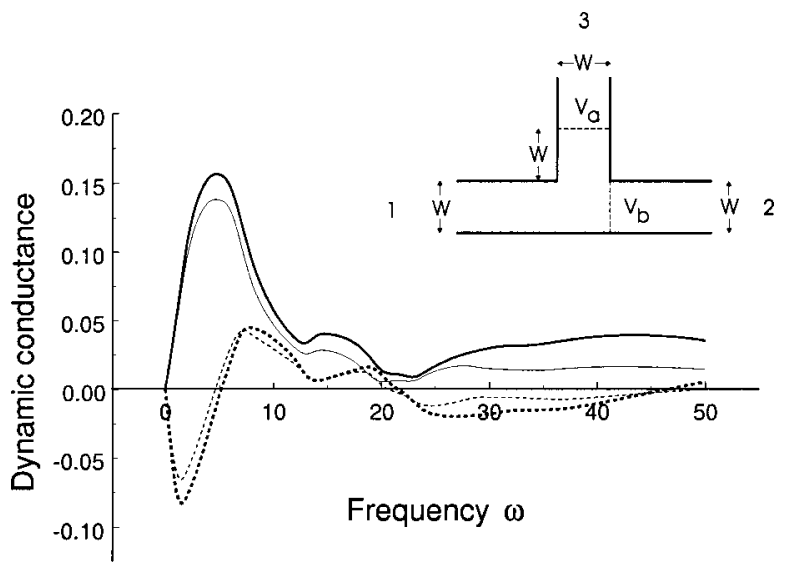

FIG. 2. Dynamic conductance vs frequency at a fixed energy $E=16.43$ for a 2D T-shaped waveguide. This figure also shows a comparison of dynamic conductance formulas: thick lines for Eq. (1) of NEGF; and thin lines for Eq. (12) of SMT. Inset: The geometry of 2D electron waveguides.

The depletion of antiresonances is clearly observed in Fig. 1, where $G_{R}$ becomes nonzero as the frequency $\omega$ is increased. Figure 2 depicts the dynamic conductance of the T-shaped waveguide as a function of frequency $\omega$ for energy fixed at the first antiresonance, $E_{r}=16.43$ (see Fig. 1). The solid and dotted curves represent the dissipative and nondissipative components $G_{R}$ and $G_{I}$, respectively. Again, Fig. 2 shows $G_{R} \sim G_{I} \sim 0$ near $\omega \sim 0$ due to antiresonance and a capacitive-like dynamic response. As the frequency increases, electrons can absorb photons of energy $\omega$ and traverse the waveguide with a higher energy, $E_{r}+\omega$. When $\omega \sim 5$, the electron energy, $E_{r}+\omega$, is near the transmission plateau where transmission coefficient $T \approx 1$, which gives the electron a maximum dc conductance of $e^{2} / h$. The first peak of $G_{R}$ in Fig. 2 indicates this. Up to this frequency $G_{I}$ is negative, indicating a capacitive-like response where current follows voltage in phase. As one increases frequency further, Fig. 2 shows that transmission decreases and an inductivelike response takes over in which voltage follows current. This is because the probability of the photon-assisted process is inversely proportional to frequency. When the frequency is near $\omega=13$, dynamic conductance reaches a minimum due to the fact that $E_{r}+\omega$ is close to the second antiresonant state. In general, $G_{R}$ decreases with $\omega$ within the first subband energy range. However, when the frequency is larger than $\omega=22, E_{r}+\omega$ is greater than the second subband threshold, hence, two conducting channels cause $G_{R}$ to increase around $\omega=22$ as shown in Fig. 2. The results of Fig. 2 allow us to conclude that the dynamic response of electron waveguides is dominated by photon-assisted transport.

It is interesting to numerically examine the two terms of dynamic conductance $G_{\alpha \beta}(\omega)$ of Eq. (1). As discussed in Ref. 20 there are two contributions to dynamic conductance. First, there is a contribution directly due to the external ac perturbation, which is the first term on the right-hand side of Eq. (1); we denote this term by $G^{\mathrm{ext}}(\omega)$. Second, there is a contribution from the internal dynamic response to the external ac field, which is the second term of Eq. (1); we denote this term by $G^{\text {int }}(\omega)$. Both $G^{\text {ext }}(\omega)$ and $G^{\text {int }}(\omega)$ are complex quantities. Importantly, we note that $G^{\text {int }}(\omega)$ includes the
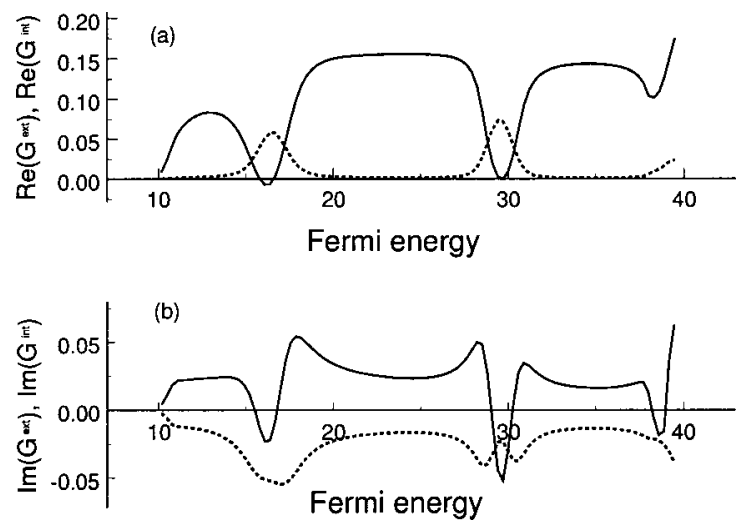

FIG. 3. The external and internal contributions to dynamic conductance for a 2D T-shaped waveguide. (a) $\operatorname{Re}[G]$ and (b) $\operatorname{Im}[G]$.

displacement current contribution to the dynamic conductance. In Fig. 3(a) we plot the real part of $G^{\text {ext }}(\omega)$ and $G^{\text {int }}(\omega)$, and in Fig. 3(b) their imaginary part, for $\omega=1.0$. The bold solid and dotted lines represent external and internal responses respectively. From Fig. 3(a), which shows the dissipative component (real part) of the dynamic conductance, we immediately conclude that external and internal responses have very different behavior: $G_{R}^{\mathrm{ext}} \equiv \operatorname{Re}\left[G^{\mathrm{ext}}(\omega)\right]$ resembles the shape of the transmission curve (see Fig. 1) with some small deviation near antiresonances; the behavior of $G_{R}^{\text {int }} \equiv \operatorname{Re}\left[G^{\text {int }}(\omega)\right]$ is very similar to that of electron dwell time for this waveguides. ${ }^{13}$ This behavior is qualitatively understandable from the point of view that both internal response and dwell time are related to the local density of states of the scattering region. Both Figs. 3(a) and 3(b) show that the external and internal contributions compensate each other in that the minimum of external contribution corresponds to the maximum of the internal contribution, a result due to induction which seeks to oppose the external change.

For the L-shaped waveguide, the dynamic conductance behaves in essentially the same manner as that of the T-shaped system, namely dominated by antiresonances. For instance, Fig. 4 shows the results for the L-shaped structure, with $G_{\alpha \beta}(\omega)$ versus Fermi energy for different frequencies.

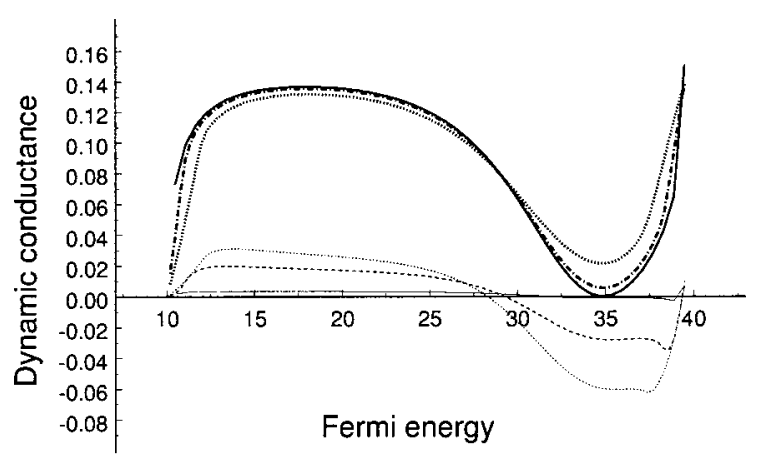

FIG. 4. The dynamic conductance as a function of Fermi energy for an L-shaped waveguide. The results are obtained by using the NEGF. Here the upper three lines (solid, dashed, and dotted) show the real part of the dynamic conductance, while the lower three lines (thin solid, dashed, and dotted) represent the imaginary part of the dynamic conductance with $\omega$ $=0.1,1.0$, and 2.0. 


\section{DISCUSSION AND SUMMARY}

The calculation reported in the previous section was carried out by evaluating nonequilibrium Green's functions and using the NEGF dynamic theory. ${ }^{20}$ We point out that there is another way to study dynamic conductance when a system is near equilibrium. Büttiker, et al. have derived ${ }^{11}$ a formula for dynamic conductance which, through the self-consistent evaluation of an internal potential response to the external ac perturbation, effectively included contributions from the displacement current. Their approach is from the scattering matrix point of view and is also easily applicable to practical calculations such as reported here. Their formula is expressed as

$$
g_{\alpha \beta}^{I}=g_{\alpha \beta}(\omega)-\frac{\sum_{\gamma} g_{\alpha \gamma}(\omega) \Sigma_{\delta} g_{\delta \beta}(\omega)}{\sum_{\gamma \delta} g_{\gamma \delta}(\omega)},
$$

where

$$
\begin{aligned}
g_{\alpha \beta}(\omega)= & \frac{e^{2}}{h} \int d E \operatorname{Tr}\left[1_{\alpha} \delta_{\alpha \beta}-s_{\alpha \beta}^{\dagger}(E) s_{\alpha \beta}(E+\hbar \omega)\right] \\
& \times \frac{f(E)-f(E+\hbar \omega)}{\hbar \omega} .
\end{aligned}
$$

In this SMT equation, the external response $g_{\alpha \beta}(\omega)$ due to an ac field is defined as the response of the system for a fixed electrostatic potential.

Equation (12) can be easily evaluated if one has the scattering matrix $s_{\alpha \beta}$. Near an antiresonant point $E=E_{r}$, the scattering matrix for a symmetric system can be approximated by the Breit-Wigner formula: ${ }^{31} \quad s_{\alpha \beta}=1-\delta_{\alpha \beta}$ $-i \Gamma / 2 /(\Delta E+i \Gamma / 2)$ where $\Delta E=E-E_{r}$. From Eq. (12), we obtain $g_{12}^{I} \equiv X_{1}+i X_{2}$ with

$X_{1}=\frac{e^{2} \Gamma}{4 h \hbar \omega}\left[\frac{4 \omega}{\Gamma}+\arctan \left(\frac{\Delta E+\omega}{\Gamma / 2}\right)-\arctan \left(\frac{\Delta E-\omega}{\Gamma / 2}\right)\right]$

and

$X_{2}=\frac{e^{2} \Gamma}{8 h \hbar \omega} \ln \left[\frac{(\Delta E+\omega)^{2}+(\Gamma / 2)^{2}}{(\Delta E)^{2}+(\Gamma / 2)^{2}} \frac{(\Delta E-\omega)^{2}+(\Gamma / 2)^{2}}{(\Delta E)^{2}+(\Gamma / 2)^{2}}\right]$.

In the present context of dynamic response of electron waveguides, we have numerically confirmed that the two formula (1) and (12) give qualitatively exact and quantitatively very close results. In fact it can be verified straightforwardly that the NEGF formulation and Eq. (1) reduce to Eq. (12) if one neglects nonequilibrium quantities such as $g^{r, a}$ and $\sigma^{r, a}$. Figure 2 shows a quantitative comparison of $G_{\alpha \beta}$ as a function of $\omega$; the agreement is good. There have been detailed discussions of the formal relation between linear response theory (such as SMT) and the NEGF theory. ${ }^{23,24}$ Figure 2 gives a direct numerical comparison for $2 \mathrm{D}$ waveguides in this regard. We have checked numerically that the deviation between the two approaches mainly comes from the imaginary parts of their external response.

To summarize, we have investigated the dynamic conductance of electron waveguides in general terms of the ac frequency $\omega$. We have applied the theoretical formalism of NEGF, which includes electrodynamic effects to calculate
$G_{\alpha \beta}(\omega)$ such that it also includes contributions from the displacement current. From the results, we conclude that variations of $G_{\alpha \beta}(\omega)$ as a function of electron energy are dominated by antiresonances at small values of $\omega$, but these antiresonances are smeared out when $\omega$ increases. Since an antiresonance is caused by a quasibound state, the behavior of $G_{\alpha \beta}(\omega)$ suggests a way to experimentally probe quasibound states in these open waveguides. By steadily varying an applied voltage through the connecting probes, one finds quasibound states of that system measuring its capacitivelike response: an abrupt change in its value shows the existence of a quasibound state. For the electron waveguides studied here, it is the antiresonance which is responsible for a change of dynamic response from capacitive-like to inductive-like behavior and vice versa. Our results show clear evidence of photon-assisted transport as $\omega$ is varied. Essentially, an electron can absorb a photon thus sampling a higher energy band where transport behavior is different. This process is indicated by the oscillatory behavior of $G_{\alpha \beta}(\omega)$ as a function of $\omega$ at a fixed electron Fermi energy. One further interesting behavior is the fact that external and internal contributions to $G_{\alpha \beta}(\omega)$ are always opposing each other: as one increases the other decreases. This is easily understood because the internal contribution is due to induction caused by the external ac perturbation.

\section{ACKNOWLEDGMENTS}

The authors thank Dr. T. Boyce for critical reading of the manuscript. The authors gratefully acknowledge support by RGC Grant No. HKU7115/98P, and a CRCG grant from the University of Hong Kong. H. G. is supported by the NSERC of Canada and FCAR of Québec. The authors thank the Computer Center of the University of Hong Kong for computational facilities.

${ }^{1}$ F. Sols et al., Appl. Phys. Lett. 54, 350 (1989).

${ }^{2}$ J. A. del Alamo and C. C. Eugster, Appl. Phys. Lett. 56, 78 (1990); C. C. Eugster and J. A. del Alamo, Phys. Rev. Lett. 67, 3586 (1991).

${ }^{3}$ N. Tsukada et al., Appl. Phys. Lett. 56, 2527 (1990).

${ }^{4}$ J. Wang et al., Appl. Phys. Lett. 59, 3075 (1991).

${ }^{5}$ Y. Hirayama et al., Phys. Rev. B 48, 7991 (1993).

${ }^{6}$ R. A. Webb et al., Phys. Rev. B 37, 8455 (1988).

${ }^{7}$ J. B. Pieper and J. C. Price, Phys. Rev. Lett. 72, 3586 (1994).

${ }^{8}$ P. G. N. de Vegvar et al., Phys. Rev. B 38, 4326 (1988).

${ }^{9}$ W. Chen et al., Phys. Rev. Lett. 73, 146 (1994).

${ }^{10}$ T. H. Oosterkamp et al., Phys. Rev. Lett. 78, 1536 (1997).

${ }^{11}$ M. Büttiker et al., Phys. Rev. Lett. 70, 4114 (1993).

${ }^{12}$ Z. S. Ma et al., Phys. Rev. B 59, 7575 (1999).

${ }^{13}$ J. Wang and H. Guo, Phys. Rev. B 54, R11090 (1996).

${ }^{14}$ S. Datta, Superlattices Microstruct. 6, 83 (1989); F. Sols et al., J. Appl. Phys. 66, 3892 (1989); L. F. Register et al., ibid. 69, 7153 (1991); J. Wang et al., ibid. 75, 2721 (1994); C. H. Yiu and J. Wang, ibid. 80, 4208 (1996).

${ }^{15}$ J. Wang and H. Guo, Appl. Phys. Lett. 60, 654 (1992).

${ }^{16}$ R. L. Schult et al., Phys. Rev. B 39, 5476 (1989); M. M. Dignam et al., ibid. 49, 2269 (1994).

${ }^{17}$ W. Porod et al., Appl. Phys. Lett. 61, 1350 (1992); Z. Shao et al., Phys. Rev. B 49, 7453 (1994)

${ }^{18}$ P. J. Price, Appl. Phys. Lett. 62, 289 (1993); Phys. Rev. B 48, 17301 (1993); E. Tekman and P. F. Bagwell, ibid. 48, 2553 (1993); J. Wang et al., Appl. Phys. Lett. 65, 1793 (1994).

${ }^{19}$ E. Emberly and G. Kirczenow, Phys. Rev. Lett. 81, 5205 (1998).

${ }^{20}$ B. G. Wang et al., Phys. Rev. Lett. 82, 398 (1999).

${ }^{21}$ Gauge invariance condition simply means that the electric current passing 
through the system cannot depend on the choice of zero potential. See, for example, Ref. 22.

${ }^{22}$ M. Büttiker and T. Christen, in Quantum Transport in Semiconductor Submicron Structures, edited by B. Kramer (Kluwer Academic, Dordrecht, 1996), pp. 263.

${ }^{23}$ A. P. Jauho, N. S. Wingreen, and Y. Meir, Phys. Rev. B 50, 5528 (1994).

${ }^{24}$ M. P. Anantram and S. Datta, Phys. Rev. B 51, 7632 (1995).

${ }^{25}$ M. J. McLennan, Y. Lee, and S. Datta, Phys. Rev. B 43, 13846 (1991).

${ }^{26}$ S. Datta, Electronic Transport in Mesoscopic Systems (Cambridge University Press, New York, 1995).

${ }^{27}$ D. S. Fisher and P. A. Lee, Phys. Rev. B 23, 6851 (1981).

${ }^{28} \mathrm{M}$. Büttiker and T. Christen, in Mesoscopic Electron Transport, NATO
Advanced Study Institute, Series E: Applied Science Vol. 345, edited by L. L. Sohn, L. P. Kouwenhoven, and G. Schoen (Kluwer Academic, Dordrecht, 1997), p. 259.

${ }^{29}$ M. Büttiker, H. Thomas, and A. Prétre, Z. Phys. B: Condens. Matter 94, 133 (1994).

${ }^{30}$ M. Büttiker, A. Prétre, and H. Thomas, Phys. Rev. B 54, 8130 (1996).

${ }^{31}$ We should mention that the simple model used in Ref. 17 for a T-shaped junction is useful only in explaining dc conductance. When expanding $s_{11}$ and $s_{12}$ near the antiresonant point $k L=\pi$, their expression gives $s_{11}=$ $-1 /\left(1+i L^{2} \Delta E / \pi\right)$ and $s_{12}=1-1 /\left(1-i L^{2} \Delta E / \pi\right)$. This satisfies $s_{12}^{*}$ $-s_{11}=1$ which is different from the usual Breit-Wigner form for symmetric system $s_{12}-s_{11}=1$. Because of this, it incorrectly predicts inductive-like behavior near the antiresonant point. 